\title{
LETTERS
}

consent was gained from her family or team members. Unless the patient has executed a lasting power of attorney (LPA) whereby a patient nominates a chosen donee (or donees) to make decisions on his or her behalf then nobody but that patient may consent to medical treatment. The authors make no reference to an LPA existing. When consent cannot be gained, it is for the clinicians involved to act in that patient's best interests; acting in a non-competent patient's best interests is not a tautology for gaining consent. This might been seen by many to be focusing too much on minutiae, but as the law is heavily dependent upon the words, terms and phrases used, it becomes increasingly important to ensure these are correct.

A. C. L. Holden

1. Stagnell S, Burrows G. Cojoined cementoma. Br Dent J 2013; 215: 267.

2. Mental Capacity Act 2005 s.1(5)

Drs Stagnell and Burrows respond: We wish to acknowledge the response to our original publication: we would like to make clear, that all guidelines are followed routinely whilst engaging in clinical activity with particular reference to the Mental Capacity Act and matters of consent. In this case, the patient's daughters did carry LPA, and we appreciate it was an oversight to have not made reference to this in our initial article and we apologise for any confusion or concern caused.

DOI: 10.1038/sj.bdj.2013.1197

\section{IT'S BEEN SAID, STUPID}

Sir, on reading your timely editorial, It's the environment, stupid, ${ }^{1}$ I paused on your comment that 'environmental issues in dentistry, and indeed medicine, have received scant attention.... I did this as lead author of a paper entitled Dental practice and the environment, ${ }^{2}$ published in 1998. This paper, which summarises the issues considered to be pertinent to the environmental effects of the clinical practice of dentistry in the 1990s, stemmed from a lecture I presented at the Silver Jubilee Meeting of the British
Society for Restorative Dentistry, held in 1993. The lecture was entitled Are dentists an environmental hazard? To the best of my knowledge, Professors Mjør and Bellinger (an environmental scientist) and I were on our own at the time in publishing on the subject of dental practice and the environment.

The world has moved on since the publication of that paper but I would suggest that much of its content remains relevant today. Materials and agents used in the clinical practice of dentistry which may pose a hazard to the environment include anaesthetic gases (also gases used in conscious sedation), base metal debris, disinfectants, etchants, monomers (ie initiators, accelerators, inhibitors, stabilisers, primers and conditioners) and associated reagents, clinical waste, X-ray processing solutions and drugs, including antibiotics. With the growing popularity of procedures such as tooth bleaching, which relies on the action of one or more reducing agents, and the increasing use of 'bioactive' materials in dentistry, the list of dentally related hazards to the environment, in my opinion, is no longer complete. As such, much-needed, would be 'champions of green thinking' in dentistry need to think much more widely than indicated in your editorial. Critically, in environmental auditing, it is important to adopt a 'cradle to grave' approach as, only by considering the sourcing of raw materials and the manufacturing, use and eventual disposal of consumables, devices and equipment is it possible to define the "environmental footprint' of an activity. Such thinking is clearly behind the Minimata Convention which requires dentistry to phase down the use of dental amalgam.

As discussed in my earlier paper ${ }^{2}$ we must not expect individual countries or regions to realise environmental goals and responsibilities by imposing costly additions to existing regulation. The environmental impact of dentistry may be relatively small but this does not exonerate anyone from critically reviewing and reducing the environmental impact of their clinical practice. As with recycling, switching off unnecessary lighting, turning down the heating thermostat and not running water while brushing your teeth, if everyone in the profession did something, the overall effect would be substantial. Needless to say, the safety of patients must never be compromised in making the practice of dentistry more environmentally friendly. As in all matters in clinical practice, the interests of the patient come first.

\section{N. Wilson, by email}

1. Hancocks S A. It's the environment, stupid. Br Dent J 2013; 215: 375.

2. Wilson N H F, Bellinger E G, Mjor I A. Dental practice and the environment. Int Dent J 1998; 48: 161-166.

DOI: 10.1038/sj.bdj.2013.1198

\section{EXUBERANT HYPERCEMENTOSIS}

Sir, Stagnell and Burrows report a case of 'cojoined [sic] cementoma' affecting the maxillary central and lateral incisors (BDJ 2013; 215: 267). The racial origin of the patient, presence or absence of Paget's disease of bone and the vitality status of the affected teeth are not specified. 'Cementoma' is not recognised by the World Health Organisation in its latest classification of odontogenic tumours ${ }^{1}$ and the maxillary incisors are very unusual sites for any of the cementogenic entities it currently lists. The case in point is likely to represent exuberant hypercementosis associated with non-vital, functionless teeth, ie a reactive rather than neoplastic process which was the result of long-term periapical inflammation. However, the history of oral bisphosphonate therapy is intriguing. One might speculate on its role in producing the pathological changes which, in turn, caused the surgical difficulties described and it is unfortunate histological data were also not included.

\section{A. W. Barrett, East Grinstead}

1. Barnes L, Eveson J W, Reichart P, Sidransky D (eds). World Health Organization classification of tumors: pathology and genetics of head and neck tumors. Lyon: IARC Press, 2005.

DOI: 10.1038/sj.bdj.2013.1199 